\title{
Human lumbar apophyseal joint damage and intervertebral disc degeneration
}

\author{
M W Swanepoel, L M Adams, J E Smeathers
}

\begin{abstract}
Objectives-To record the extent and location of lumbar apophyseal cartilage damage, and to ascertain if the extent of damage is correlated with the grade of disc degeneration, age, or both.

Methods-The extent and location of fibrillated areas of the apophyseal cartilage of the joint surfaces of 29 lumbar motion segments were examined using computer aided image processing of Indian ink stained areas, and degeneration of the associated intervertebral discs graded using the method of Nachemson.
\end{abstract}

Results-It was found that these joints showed a greater extent and prevalence of cartilage fibrillation than the knee, hip or ankle, with significant damage in specimens younger than 30 years. Damage was predominantly located peripherally, superiorly, and posteriorly in the concave superior apophyseal surfaces, and was predominantly peripheral and posterior in the inferior surfaces, with a tendency to be located inferiorly. There was a weak correlation between apophyseal joint damage and the intervertebral disc degenerative grade, but this was inconclusive, as both increased with age. Conclusions-The pattern of damage exhibited by superior joint surfaces is most probably caused by tension on collagenous joint capsule fibres which insert into the surfaces posteriorly, so producing an area of fibrocartilage unsuited to loadbearing. Tension on such fibres would be greatest during spinal flexion. The pattern of damage of the inferior surfaces lends some support to the hypothesis that their apices impact the laminae of the lumbar vertebra inferior to them, consequent upon the degeneration and narrowing of the associated intervertebral disc. The predominantly peripheral location of fibrillation of both superior and inferior surfaces may be associated with inadequate mechanical conditioning of marginal joint areas. Disc degeneration cannot be the initial cause of apophyseal fibrillation in most specimens. The study indicates a need for regular spinal exercise, starting at a young age.

(Ann Rheum Dis 1995; 54: 182-188)

The apophyseal joints have been suggested to cause pain; possible sources include pinching of synovial folds between the joint surfaces, ${ }^{1-3}$ excessive stretching of the joint capsule, ${ }^{4}$ impingement of the tips of the inferior apophyses of a lumbar vertebra against the laminae of the neighbouring inferior vertebra, ${ }^{45}$ and compression of the spinal nerve roots as a result of stenosis of the intervertebral foramina caused by apophyseal osteophytosis. ${ }^{4}$ Clinical evidence exists to support the hypothesis of an apophyseal origin for pain, ${ }^{6-9}$ and nerves likely to be nociceptive in function have been identified in capsular tissue. ${ }^{10-13}$

Putti $^{14}$ noted extreme asymmetry, osteoarthrosis, and ankylosis of apophyseal joints. Apophyseal joint orientation has been suggested to influence intervertebral disc pathology. ${ }^{1516}$ Ghormley $^{5}$ believed that apophyseal osteoarthrosis could cause pain. Putti and Logroscino ${ }^{17}$ ascribed apophyseal osteoarthrosis to irregularities of joint shape and motion. Marginal apophyseal fractures have been noted. ${ }^{5}{ }^{18-20}$ Ghormley ${ }^{5}$ and Mitchell ${ }^{20}$ noted the difficulty of diagnosing such fractures.

Butler et $a l,{ }^{21}$ who examined intervertebral discs by magnetic resonance imaging (MRI) and the associated apophyseal joints with computed tomography (CT), concluded that degeneration of the intervertebral disc invariably precedes that of the apophyseal joints. This conclusion may be wrong if MRI was better at detecting disc degeneration than CT was at detecting apophyseal osteoarthrosis; apophyseal joint damage has been found independently of degeneration of the intervertebral disc and body, in both mediaeval skeletons $\mathrm{s}^{22}$ and modern specimens. ${ }^{2324}$

A study of the extent and location of apophyseal cartilage damage should reveal much about its pathogenesis and interaction with intervertebral disc degeneration. This paper presents such an investigation-one component of a study which also examined the compressive modulus and thickness of lumbar apophyseal cartilage, and the joint morphology. It aimed to ascertain if the extent of apophyseal cartilage damage is correlated with disc grade, age, or both, and to record the distribution of damage to the apophyseal joint surfaces.

Material and methods

Thirty one upper lumbar motion segments (table 1) were dissected and the lumbar apophyseal surfaces stained with Indian ink, following the method of Meachim..$^{25} \mathrm{~A}$ drawing and photographic record were made of each joint surface. The intervertebral discs were 
sectioned in the transverse plane, photographed, and their degenerative state graded using the method of Nachemson. ${ }^{26}$

The photographic slides taken of the joint surfaces during dissection were projected onto a screen and tracings made of the outlines of the entire joint surfaces. Those areas stained with Indian ink, indicating fibrillation, were marked and cross-hatched. Damage was considered to be any disruption of the cartilage worse than slight flecking. This was subjective, but damaged areas were selected by the same individual for all the joint surfaces. Transparent acetate sheets were placed over the tracings and two black silhouettes made of each joint surface; the first being of the entire joint surface and the second of the damaged areas alone. The silhouettes were marked to indicate anatomical orientation.

The silhouettes were placed sequentially under a low magnification dissecting microscope (Type 162065, Wild, Heerbrugg, Switzerland). The image was manually adjusted by varying the intensity of the transmitted light. A closed circuit television camera (HV-720K, Hitachi Denshi, Japan) transmitted the images to an image processing unit (Image Manager, Sight Systems Ltd, Newbury, Berks, UK) attached to a desktop computer (Elonex PC386 SXM/16, Elonex plc, 2 Apsley Way, London NW2 7LF). The image was reproduced on a CRT display (Philips CM 8833/05G) in 256 shades from black to white, with a resolution of $512 \times 512$ pixels. The focused image was stored for digital manipulation and analysis. The narrow range of the black silhouettes allowed the contrast to be altered digitally to remove artefacts.

The silhouettes of each joint surface and its damaged areas were grabbed sequentially, so that the extent and distribution of damage could be measured. The area, geometric centre, mean distance at which surface area pixels were located from this centre, maximum anteroposterior length and maximum superoinferior length of the entire joint surface were calculated. The image of the damaged areas was then superimposed on the image of the entire surface, with the same magnification and alignment. The program then: (1) Calculated the ratio of the damaged area to the entire joint area. (2) Found the geometric centre of the combined damaged areas. (3) Ascertained the position of the geometric centre of the damaged areas with respect to the centre of the entire joint area in the anteroposterior and superoinferior directions. (4) Divided the distance of the centre of the damaged areas from the centre of the joint surface by the mean distance of the whole area from the centre of the joint surface. If the ratio was greater than $1 \cdot 0$, most damage was peripheral, and if less than one, it was central.

These measurements were compared not only between themselves, but also with the age of the specimens and the grade of degenerative changes of the intervertebral discs. Specimens $\mathrm{Q}$ and $\mathrm{R}$ were excluded from the analysis of the extent and location of apophyseal fibrillation because of complete intervertebral ankylosis.
For purposes of the correlation between disc grade and apophyseal fibrillation, the calcified intervertebral discs of these specimens were recorded as grade $4+$, and the apophyseal ankylosis as $100 \%$ damage.

If the extent of damage generally increased with the measure of its peripheral distribution and specimen age, then it could be concluded that damage starts centrally, spreading outwards. Similarly, it could be determined if damage occurred predominantly in a particular part of the joint surfaces.

The displacement of the geometric centre of the damaged area from the centre of the entire joint surface in the anteroposterior (AP) and superoinferior (SI) directions was expressed as ratios of the maximum AP width and SI length of the joint surface, respectively. The position of the geometric centre of the damaged area could be expressed anatomically, for example as being located anteriorly and posteriorly with respect to the centre of the joint. The automated calculations eliminated subjectivity, and the use of ratios allowed comparisons between joint surfaces regardless of differences in joint size and in the magnification incurred in photography and image grabbing.

\section{Results}

Table 1 lists details of the disc specimens. Specimens Q1 and R1 exhibited complete intervertebral ankylosis, with advanced although incomplete ankylosis of the apophyseal joints. They were included in comparisons of intervertebral disc and apophyseal joint degradation with each other and with age, but were excluded from subsequent analyses.

The percentage of area damaged was calculated as a mean value for the four

\section{Table 1 List of 31 specimens studied}

\begin{tabular}{|c|c|c|c|c|}
\hline \multicolumn{4}{|c|}{ Specimen } & \multirow[t]{2}{*}{ Cause of death } \\
\hline Code & Type & $\operatorname{Sex}$ & Age & \\
\hline B1 & $\mathrm{L} 1+\mathrm{L} 2$ & $\mathbf{M}$ & 78 & Lung carcinoma \\
\hline $\mathrm{Cl}$ & $\mathrm{L} 1+\mathrm{L} 2$ & $\mathbf{M}$ & 41 & Cardiac arrest \\
\hline D1 & $\overline{\mathrm{L}} 1+\mathrm{L} 2$ & M & 55 & Airway obstruction \\
\hline E1 & $\mathrm{L} 1+\mathrm{L} 2$ & $\mathbf{F}$ & 46 & Cardioresp. failure \\
\hline F1 & $\mathrm{L} 2+\mathrm{L} 3$ & $\mathbf{M}$ & 39 & Cardiac arrest \\
\hline G1 & $\mathrm{L} 1+\mathrm{L} 2$ & $\mathbf{F}$ & 35 & Unknown \\
\hline H1 & $\mathrm{L} 2+\mathrm{L} 3$ & $\mathbf{M}$ & 21 & RTA \\
\hline I1 & $\mathrm{L} 3+\mathrm{L} 4$ ? & $\mathbf{M}$ & 20 & Head injury \\
\hline $\mathrm{J} 1$ & $\mathrm{~L} 3+\mathrm{L} 4$ & $\mathbf{M}$ & 23 & Unknown \\
\hline K1 & $\mathrm{L} 1+\mathrm{L} 2$ & $\mathbf{M}$ & 19 & Unknown \\
\hline $\mathrm{L} 1$ & $\bar{L} 1+\bar{L} 2$ & M & 54 & Cardiac arrest \\
\hline M1 & $\mathrm{L} 1+\mathrm{L} 2$ & $\mathbf{M}$ & 27 & Neoplasm \\
\hline $\mathrm{N} 1$ & $\mathrm{~L} 1+\mathrm{L} 2$ & M & 54 & Cardiac arrest \\
\hline 01 & $\mathrm{~L} 1+\mathrm{L} 2$ & $\mathbf{M}$ & 59 & Cardiac arrest \\
\hline P1 & $\mathrm{L} 1+\mathrm{L} 2$ & $?$ & 60 & Cardiac arrest \\
\hline Q1 & $\mathrm{L} 1$ + L2 + L3 & $\mathbf{M}$ & 70 & Bladder carcinoma \\
\hline R1 & $\mathrm{T} 12+\mathrm{L} 1+\mathrm{L} 2$ & F & $60 ?$ & Cardiac arrest \\
\hline S1 & $\mathrm{L} 1+\mathrm{L} 2$ & $\mathbf{M}$ & 31 & Head injury \\
\hline $\mathrm{T} 1$ & $\mathrm{~L} 1+\mathrm{L} 2$ & $\mathbf{M}$ & 34 & Cardiac arrest \\
\hline U1 & $\mathrm{L} 1+\mathrm{L} 2$ & $\mathbf{M}$ & 35 & Cardiac arrest \\
\hline V1 & $\mathrm{L} 1+\mathrm{L} 2$ & $\mathbf{M}$ & 35 & Heart transplant \\
\hline$W_{1}$ & $\overline{\mathrm{L}} 1+\mathrm{L} 2$ & M & 35 & Cardiac arrest \\
\hline $\mathrm{X} 1$ & $\mathrm{~L} 1+\mathrm{L} 2$ & M & 30 & Mitral valve \\
\hline Y1 & $\mathrm{L} 1+\mathrm{L} 2$ & $\mathbf{M}$ & 16 & Cardiac arrest \\
\hline A2 & $\mathrm{L} 1+\mathrm{L} 2$ & F & 20 & Cardiac arrest \\
\hline B2 & $\mathrm{L} 2+\mathrm{L} 3$ & $\mathbf{M}$ & 22 & RTA \\
\hline $\mathrm{C} 2$ & $\mathrm{~L} 1+\mathrm{L} 2$ & $\mathrm{~F}$ & 36 & Cardioresp. failure \\
\hline D2 & $\mathrm{L} 1+\mathrm{L} 2$ & $\mathrm{~F}$ & 33 & Head injury \\
\hline E2 & $\mathrm{L} 1+\mathrm{L} 2$ & $\mathbf{M}$ & 25 & Multiple injuries \\
\hline F2 & $\mathrm{L} 1+\mathrm{L} 2$ & $\mathbf{M}$ & 17 & Head injury, RTA \\
\hline G2 & $\mathrm{L} 1+\mathrm{L} 2$ & $\mathbf{M}$ & 35 & Cardioresp. failure \\
\hline
\end{tabular}

Mean age 38 (SD 17) years. Heart transplant $=$ failed heart transplant; $R T A=$ road traffic accident. 
Table 2 Percentage of joint areas damaged

\begin{tabular}{llllll}
\hline $\begin{array}{l}\text { Specimen } \\
\text { code }\end{array}$ & $\begin{array}{l}\text { Superior } \\
\text { left }\end{array}$ & $\begin{array}{l}\text { Superior } \\
\text { right }\end{array}$ & $\begin{array}{l}\text { Inferior } \\
\text { left }\end{array}$ & $\begin{array}{l}\text { Inferior } \\
\text { right }\end{array}$ & $\begin{array}{l}\text { Mean all } \\
\text { areas }\end{array}$ \\
\hline B1 & 38 & 50 & 40 & 23 & 38 \\
C1 & 47 & 54 & 39 & 71 & 53 \\
D1 & 6 & 8 & 20 & 39 & 18 \\
E1 & 66 & 51 & 71 & 75 & 66 \\
F1 & 32 & 57 & 13 & 19 & 30 \\
G1 & 52 & 57 & 53 & 51 & 53 \\
H1 & 45 & 76 & 32 & 41 & 49 \\
I1 & 72 & 16 & 19 & 13 & 30 \\
J1 & 68 & 38 & 40 & 27 & 43 \\
K1 & 35 & 20 & 23 & 29 & 27 \\
L1 & 28 & 37 & 11 & 63 & 35 \\
M1 & 28 & 29 & 29 & 43 & 32 \\
N1 & 70 & 30 & 100 & 31 & 58 \\
O1 & 41 & 72 & NA & NA & 57 \\
P1 & 33 & 72 & 100 & 78 & 71 \\
S1 & 75 & 12 & 25 & 72 & 46 \\
T1 & 54 & 51 & 35 & 52 & 48 \\
U1 & 52 & 41 & 36 & 40 & 42 \\
V1 & 76 & 41 & 51 & 36 & 51 \\
W1 & 79 & 29 & 86 & 72 & 67 \\
X1 & 46 & 58 & 38 & 50 & 48 \\
Y1 & 68 & 73 & 40 & 45 & 57 \\
A2 & 46 & 46 & 45 & 27 & 41 \\
B2 & 36 & 47 & 12 & 31 & 32 \\
C2 & 36 & 45 & 36 & 50 & 42 \\
D2 & 46 & 62 & 83 & 50 & 60 \\
E2 & 80 & 52 & 41 & 19 & 48 \\
F2 & 32 & 29 & 45 & 36 & 36 \\
G2 & 8 & 10 & 77 & 51 & 37 \\
Mean (SD) & $48(20)$ & $44(19)$ & $44(25)$ & $44(18)$ & $45(13)$ \\
\hline & & & & &
\end{tabular}

$\mathrm{NA}=$ Data not available.

apophyseal joint surfaces of each lumbar motion segment. Table 2 lists the percentage of joint areas found to be at least slightly fibrillated: each type of surface, whether superior, inferior, left or right, had a mean extent of damage of $44-48 \%$. The extent of damage of contralateral inferior, convex joint surfaces was significantly correlated (mean surface damage on the left $44 \cdot 2$ (SD $24 \cdot 7) \%$; on the right $\left.43.9(17.9) \% ; r^{2}=0.16, p<0.05\right)$, but this was not true of any other surfaces. There was a weak correlation between the extent of apophyseal joint damage and donor age (fig 1), and a strong correlation between the degenerative grade of the intervertebral

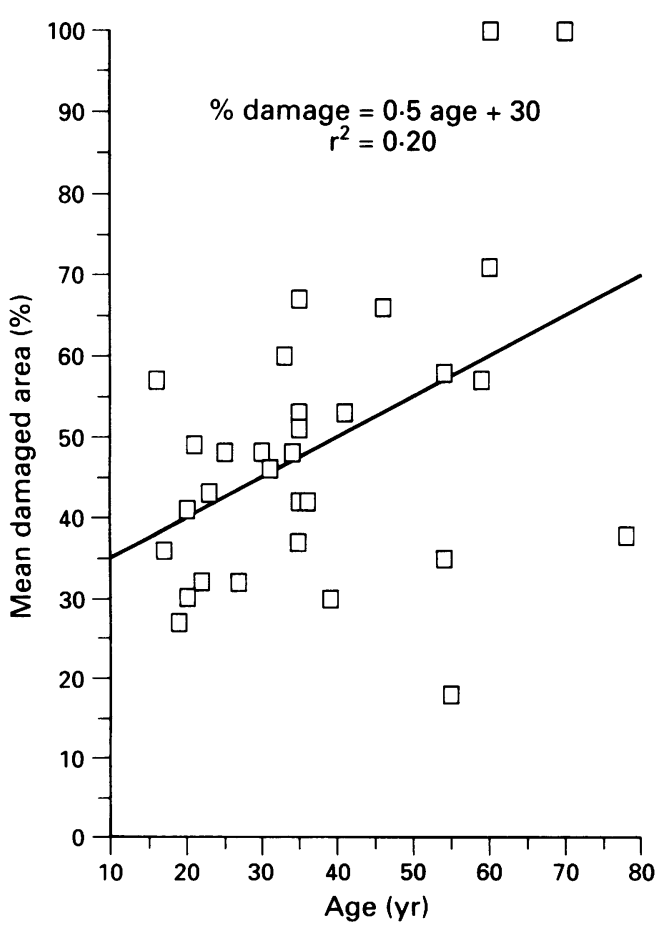

Figure 1 Variation of the extent of apophyseal joint damage with age (mean damaged area of the four joint surfaces of each specimen).

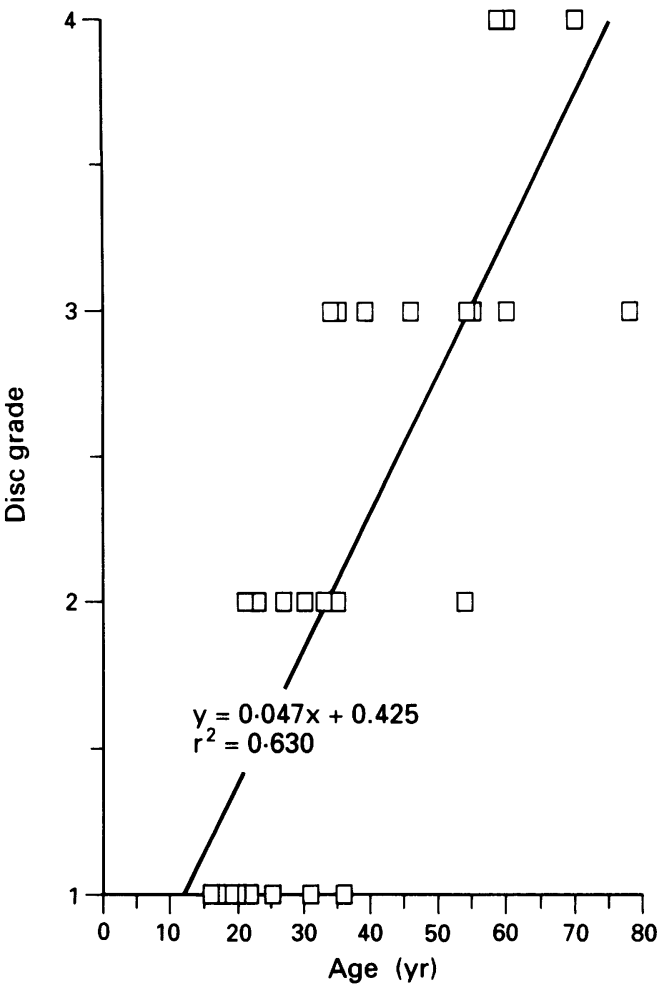

Figure 2 Variation of the state of the intervertebral discs with age. Discs graded on a scale of $1=$ intact, to $4=$ most degenerate.

discs and age (fig 2), so that it is unsurprising that the extent of lumbar apophyseal joint damage was correlated with the grade of the intervertebral discs (fig 3).

Table 3 summarises the locations of centres of joint damage. The centres of the damaged areas were found to be posterior to the centres of the concave superior joint surfaces, although this was no more than a tendency in the case

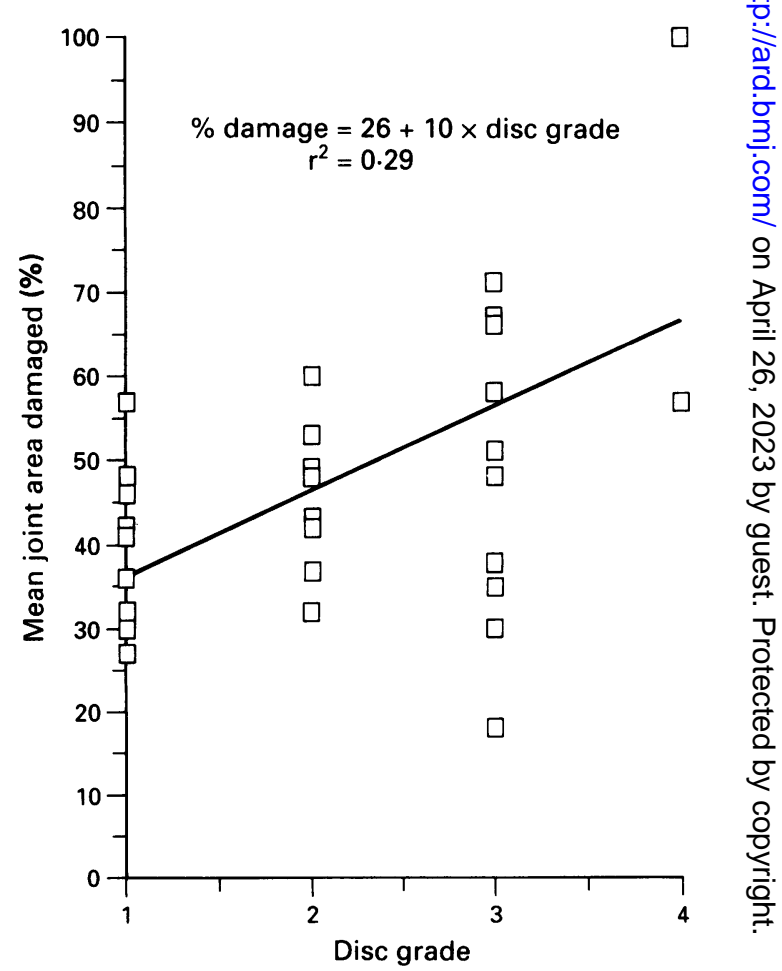

Figure 3 Variation of the extent of apophyseal joint fibrillation with disc grade ( $1=$ intact $; 4=$ most degenerate). 
Table 3 Anteroposterior, superoinferior and central-peripheral locations of the centre of joint damage

\begin{tabular}{|c|c|c|c|c|}
\hline Foint surface & Ratio (SD) & Location & $t$ & $p$ \\
\hline \multicolumn{5}{|c|}{ Anteroposterior location (Ratio values: $0=$ Central; $+1=$ posterior; $-1=$ anterior) } \\
\hline All $(n=114)$ & $0.034(0.092)$ & Posterior & 3.92 & $<0.001$ \\
\hline Superior $(n=58)$ & $0.046(0.098)$ & Posterior & 3.57 & $<0.001$ \\
\hline Inferior $(n=56)$ & $0.021(0.086)$ & Posterior & 1.83 & NS \\
\hline \multicolumn{5}{|c|}{ Superoinferior location (Ratio values: $0=$ Central; $+1=$ inferior; $-1=$ superior) } \\
\hline All $(n=114)$ & $-0.003(0 \cdot 108)$ & Superior & $0 \cdot 30$ & NS \\
\hline Superior $(n=58)$ & $-0.028(0.102)$ & Superior & $2 \cdot 09$ & $<0.05$ \\
\hline Inferior $(n=56)$ & $0.024(0 \cdot 108)$ & Inferior & 1.66 & NS \\
\hline \multicolumn{5}{|c|}{ Central-peripheral location (Ratio values: $0=$ Central; $1 \cdot 5=$ peripheral; $1 \cdot 0=$ no bias) } \\
\hline All $(n=114)$ & $1 \cdot 100(0 \cdot 134)$ & Peripheral & $7 \cdot 97$ & $<0.001$ \\
\hline Superior $(n=58)$ & $1.086(0 \cdot 139)$ & Peripheral & $4 \cdot 71$ & $<0.001$ \\
\hline Inferior $(n=56)$ & $1.114(0 \cdot 130)$ & Peripheral & 6.56 & $<0.001$ \\
\hline
\end{tabular}

of the inferior surfaces. Damage was biased superiorly in the superior surfaces, and the inferior bias of damage of the inferior surfaces verged on significance. Damage occurred more frequently in the peripheral areas of all surfaces than one would expect by chance. The central to peripheral distribution of the centres of damaged areas with respect to the centres of the joint surface areas is presented in figure 4 .

There was no correlation between the anteroposterior location of the centres of damage of both contralateral and articulating

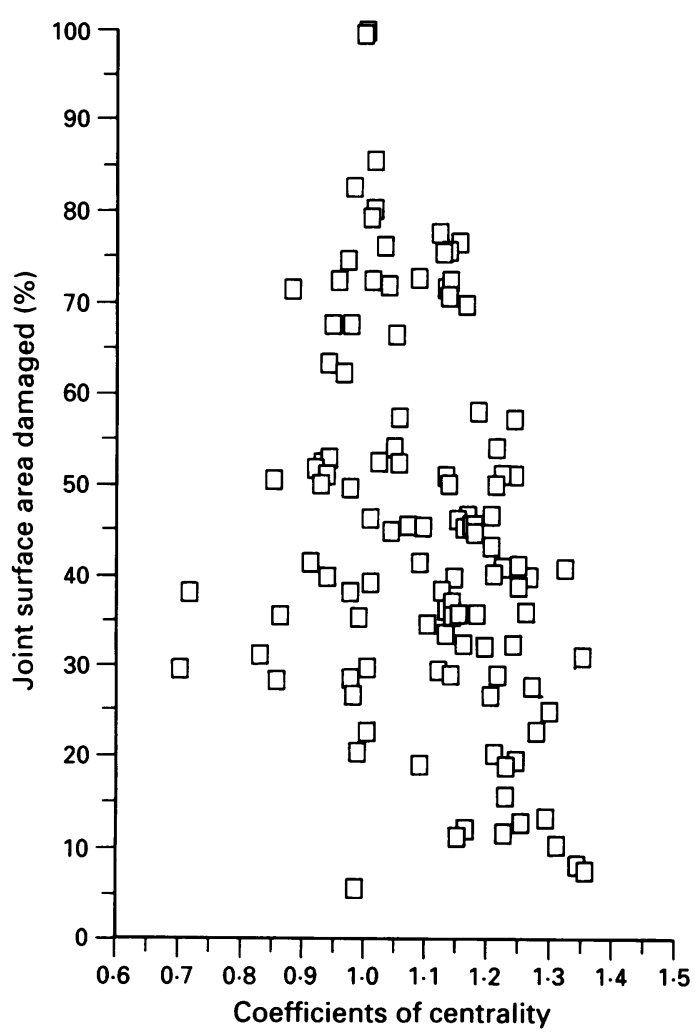

Figure 4 Variation of the central-peripheral location of joint damage with its extent. Coefficients of centrality: $0=$ Central $; 1 \cdot 0=$ no bias; $1 \cdot 5=$ peripheral .

Table 4 Significant correlations between the centrality of damage of different joint surfaces

\begin{tabular}{|c|c|c|c|c|c|}
\hline \multirow{2}{*}{$\begin{array}{l}\text { foint } \\
\text { surface }\end{array}$} & \multicolumn{2}{|c|}{ Displacement ratio } & \multirow[t]{2}{*}{$t$ test } & \multicolumn{2}{|c|}{ Correlation } \\
\hline & Left & Right & & $r^{2}$ & $p$ \\
\hline $\begin{array}{l}\text { All } \\
\text { Superior } \\
\text { Inferior }\end{array}$ & $\begin{array}{l}1 \cdot 10(0 \cdot 12) \\
1.09(0 \cdot 11) \\
1 \cdot 12(0 \cdot 12)\end{array}$ & $\begin{array}{l}1 \cdot 10(0 \cdot 15) \\
1.09(0 \cdot 16) \\
1 \cdot 11(0 \cdot 14)\end{array}$ & $\begin{array}{l}\text { NS } \\
\text { NS } \\
\text { NS }\end{array}$ & $\begin{array}{l}0.26 \\
0 \cdot 24 \\
0 \cdot 31\end{array}$ & $\begin{array}{l}<0.001 \\
<0.01 \\
<0.01\end{array}$ \\
\hline
\end{tabular}

Mean values (SD). Ratio values: $0=$ Entirely central; $1=$ no bias; $1.5=$ entirely peripheral. surfaces, but there was a correlation between the superoinferior location of the centres of damage of the contralateral inferior joint surfaces (mean displacement ratio on the left -0.031 (SD 0.107 ); on the right -0.014 $(0 \cdot 103)$ (negative values indicating inferior location); $\left.r^{2}=0.35, p<0.001\right)$. The central to peripheral damage distributions of the contralateral (concave) superior joint surfaces were correlated, as were those of the contralateral convex inferior joint surface pairs, but this was not true of the matched articulating surfaces (table 4). The extent of apophyseal cartilage damage was not correlated with the estimated cadaveric height or mass, nor with the location of the centre of joint surface damage.

\section{Discussion}

COMPUTER BASED IMAGE ANALYSIS

Computer based image analysis of Indian ink stained cartilage has not been previously attempted, thus some explanation of the method is due. Originally, slides were examined directly with the image processing unit through the 256 shade closed circuit television camera (CCTV). The contrast of the image was adjusted digitally to highlight fibrillation before the image underwent 'thresholding'; fibrillated areas stained darker than a predetermined shade were selected to be black, while the surrounding areas were set to white. Thus a binary image of the fibrillated cartilage was produced. Selection of damaged areas was unpredictable, because fibrillation was recorded uniquely within the grey scale of each slide, thus nullifying selection algorithms. This was due to:

(1) The slides being taken using a macrolens with manual aperture setting during different weather conditions, seasons, and times of day. Hence the illumination of the slides differed although they were all taken indoors with a compensatory flash.

(2) The perimeter of stained areas decreasing with an increase in the total light transmitted through non-stained areas. This effect was exacerbated by the size range of joints. (The brightness of the image had a 'washout' effect on stained areas. This problem was inherent in the use of a CCTV.)

After experimentation it was found that the analogue capability of the human eye enjoyed an advantage over the limited digital resolution of the computer. Hence the slides were projected, tracings made of the fibrillated areas (on which grades of damage were noted for future reference), and black silhouettes made from the tracings. These silhouettes made image capture in a 256 grey shade scale almost irrelevant. However, contrast stretching and thresholding, which can only be performed on grey shade scales, were retained to remove artefacts caused by optical imperfections.

It was concluded that digital image analysis is excellent for calculating lengths, areas, and orientations, and is useful for comparing photographic images taken under tightly controlled lighting, but is a poor second best 
to human judgment of shaded areas under non-uniform conditions.

JOINT DAMAGE AND DISC DEGENERATION

Is lumbar apophyseal joint damage always consequent upon intervertebral disc degeneration, as suggested by Butler et al ?21 The apophyseal cartilage surfaces were fibrillated over at least $20 \%$ of their surfaces at 20 years old, as determined by Indian ink staining (fig 1), while the intervertebral discs at the same age exhibited minimal degeneration (fig 2), so it is most unlikely that the former is invariably associated with the latter; the weak correlation between apophyseal joint damage and intervertebral disc degeneration (fig 3 ), is inconclusive. Unfortunately, the grading of intervertebral disc degeneration following the method of Nachemson ${ }^{26}$ proved to be inadequate for assessing the interplay between intervertebral disc and apophyseal joint degeneration. A biochemical analysis of the disc material, discograms, and radiographs of the vertebral endplates would have increased the objectivity and usefulness of the study.

Reduction of the intervertebral disc space has been implicated in the pathogenesis of apophyseal osteoarthrosis. ${ }^{21} 2427$ It is uncertain that fissuring and fibrosis of discs are correlated with their thickness and mechanical properties. Smeathers and Joanes ${ }^{28}$ measured the intervertebral disc space of seven upper lumbar motion segments from lateral radiographs and graded degeneration on a scale of 1-4. The negative correlation between the disc grade and intervertebral space of -0.664 indicates that disc spaces narrow with degeneration, but the probability of this being a chance trend is $0 \cdot 1$. Proof that disc space narrowing causes apophyseal osteoarthrosis would demand in vivo radiographic measurements of the same individuals over many years.

Apophyseal joint cartilage was fibrillated even when the discs were intact (fig 3). This does not contradict studies linking apophyseal osteoarthrosis to intervertebral disc degeneration, such as that of Butler et al, ${ }^{21}$ who defined osteoarthrosis to include visible loss of the joint space, and that of Lewin, ${ }^{23}$ who defined osteoarthrosis to include lesions of the subchondral bone. Several authorities ${ }^{22-24}$ are convinced that intervertebral disc degeneration cannot explain all apophyseal joint degeneration. Moreover, radiography of the apophyseal joints does not disclose cartilage damage if the joint space is preserved. ${ }^{17}$ Malmivaara et $a l^{29}$ concluded that apophyseal osteoarthrosis is unrelated to disc degeneration or vertebral body osteophytosis at the T10-L1 levels. Figures 1, 2, and 3 may indicate both that apophyseal joint cartilage softens and deteriorates with age, and that joint damage increases as a result of narrowing of the intervertebral discs. There is, however, still a wide variation in joint damage associated with grade 3 discs.

Baker et $a l^{30}$ noted that spontaneous arthrodesis/ankylosis of the apophyseal joints frequently occurs if the intervertebral bodies of the corresponding motion segments are fused, and that the first changes are fibrous ingrowth from the joint edges over the cartilage, loss of proteogylcan synthesis, and the development of a fibrous layer at the tidemark. The apophyseal joints of two specimens with calcified intervertebral discs (Q1 and R1) allowed us to confirm that arthrodesis starts at the joint margins.

Dynamic periodic loading stimulates the proteoglycan synthesis underpinning the health of articular cartilage, while prolonged or light loading has the opposite effect. ${ }^{31} 32$ It has also been shown that lumbar apophyseal joints atrophy when the spine is distracted using Harrington instrumentation; ${ }^{33}$ thus it is likely that a lack of spinal exercise because of a sedentary lifestyle would cause 'abnormal' softening of the lumbar apophyseal joint cartilage. The cephalo-caudal alignment of the upper lumbar apophyseal joints means that they experience significant loading only during spinal flexion and torsion; moreover the spinal flexion experienced by sedentary workers is of a postural rather than dynamic nature. The hypothesis that lumbar apophyseal cartilage is unusually soft is borne out by its compressive stiffness, which can be as stiff as well exercised areas of human knee cartilage, but is on average only a third of this stiffness. ${ }^{34}$ This would predispose it to mechanical disruption by infrequent high loads in the manner suggested by Seedhom et al. ${ }^{35}$

The finding that the cross-sectional area of the narrowest part of the vertebral bodies is correlated with the estimated cadaveric height, but not with the estimated cadaveric mass, coupled with the predominant cause of death, cardiac arrest, suggests that the donors we studied had not been exercising adequately. If the specimens had come from fit people, it is probable that a correlation between body weight and the lumbar vertebral cross-sectional area would exist, as height and mass would then be correlated.

The locations of damage as indicated by image processing must be carefully interpreted. Local sites of damage may not have been apparent because their influence on the overall centres and distributions of damage may have been balanced by other local areas of damage elsewhere on the joint surfaces. Thus it is likely that damage to the inferior apices of the inferior apophyses would have been significant in this study but for the inclusion of random sites of localised damage within the programming algorithm.

The tips of the inferior apophyses exhibited irregular cartilage margins and occasional gross fibrillation. Narrowing of the intervertebral disc space causes the tip of the inferior apophyses to press against the laminae of the inferior vertebra of each lumbar motion segment, especially in lumbar extension. ${ }^{27}$ It may be expected that the large tip pressures then cause irreversible damage, the production of debris and subsequent erosion of the joint surfaces by wear, together with osteophytic growth. There were specimens suggestive of this mechanism. 
The posterior and peripheral staining of the superior concave surfaces was associated both with overt fibrillation and with the fibrocartilaginous transition zone between the capsule and the articular cartilage (a zone also noted by Bogduk and Twomey ${ }^{36}$ ). The collagen fibres align with the tension exerted through the capsule, leading to fine splits between and parallel to the fibres. This would cause an initial loss of the load bearing properties of the surface, as the retention of water responsible for the resilience of the cartilage cannot be maintained. Local disruption of the articular surface would ensue, leading to the posterior fibrillation typical of superior joint surfaces. Although this was a 'normal' state, it cannot be a healthy one. It may be caused by, and would certainly be aggravated by, prolonged lumbar flexion, which subjects the apophyseal joint capsule to traction. Prolonged flexion would typically be associated with an incorrect seating posture, with loss of the lumbar lordosis.

However, this is not the only possible reason for posterior fibrillation of joint surfaces. The apophyseal joints taper slightly inwards inferiorly. ${ }^{37}$ When the narrower inferior parts of the convex inferior surfaces are drawn superiorly relative to the superior surfaces during lumbar flexion, the inferior surfaces are free to move so that their more coronal anterior parts are loaded. If, however, the surfaces do not contact each other anteriorly because of joint incongruency, then the anterior shear loading incurred between the lumbar vertebrae during flexion must be borne by the perisagittal posterior sections of the apophyseal joints. The perisagittal orientation will cause high compressive stresses between the articulating posterior joint surfaces in order to carry the anteriorly directed force-this may be described as 'wedging'. Maintenance of wedging pressures resulting from prolonged lumbar flexion would lead to contact between the collagen fibres of the articulating surfaces, and hence posterior fibrillation of the apophyseal joints.

The apophyseal surfaces of specimen L1 curved medially and anteriorly so much that their most medial borders pointed posteriorly and medially away from the coronal plane. This could be ascribed not only to the subchondral bone shape, but also to the cartilage which was wrapped around the edges of the inferior surfaces to form 'bumpers' ('fenders'), as described by Bogduk and Twomey. ${ }^{36}$ Bogduk and Twomey thought that stressing of the edges of articular processes during torsion induces bumpers to form, while Hadley $^{2}$ felt that recurrent impaction of the apices of the inferior apophyses could lead to bumpers forming inferiorly.

The cumulative incidence of apophyseal joint injury should increase with age, as the duration of the exposure to risk increases. Two injuries were noted. A crack emanated upwards from the posterior and inferior margin of the superior surface of specimen $\mathrm{H} 1$, and an inferior apophysis of specimen I1 exhibited a posterior marginal fracture ascribable to torsion. Both were old injuries. Surprisingly, the surfaces were less fibrillated than average. These cases, together with the high incidence of cartilage fibrillation in the absence of apophyseal fractures, prove that trauma plays a minor role.

Gouge marks on the surface of one superior (concave) joint surface suggested that the boney tip of the inferior apophysis of the joint had been lifted from its inferior position, and pressed into the cartilage surface. This would have occurred as a result of combined lumbar flexion and torsion.

In view of the extent and incidence of lumbar apophyseal joint fibrillation, and the softness of the cartilage, it is suggested that the apophyses are deliberately loaded by twisting the trunk around the cephalo-caudal axis in the standing posture several times each day. This activity should ensure adequate mechanical stimulus for healthy conditioning of the cartilage. Further, it is suggested that prolonged lumbar flexion is avoided to minimalise large static stresses caused by wedging of more sagittally orientated apophyseal joints, and to reduce tension on the insertions of the joint capsules into the concave superior joint surfaces posteriorly.

\section{Conclusions}

All the apophyseal joints in this study ranging in age from 16 to 78 years showed signs of cartilage fibrillation. The prevalence of fibrillation in the lumbar apophyseal joints is greater than that of the knee, hip or ankle. On average $45 \%$ of the total joint surface was damaged.

Damage of the superior, concave apophyseal joints was predominantly peripheral, superior, and posterior, that of the inferior apophyseal joints was predominantly peripheral and posterior.

The pattern of damage suggests that it is initiated in areas of cartilage which are soft, either as a result of a lack of adequate loading, or because of their location close to the insertion of collagenous joint capsule fibres.

There is a weak correlation between the amount of apophyseal joint damage and the intervertebral disc degenerative grade $\left(r^{2}=0 \cdot 29\right)$, both of which increase with age.

1 Keller G. Die Arthrose der Wirbelgelenken in ihre Beziehung zum Ruckenschmerz. Z Orthop Ihre Grenzgeb 1959; 91: 538-50.

2 Hadley L A. Anatomico-roentgenographic studies of the postero-spinal articulations. Am $\mathcal{F}$ Roent 1961; 86: 270-6. $3 \mathrm{Kos} J$, Wolf J. Les menisques intervertebraux et leur role possible dans les blocages vertebraux. Ann Med Phys 1972; 15: 203-17.

4 Hadley L A. Subluxation of the apophyseal articulations with boney impingement as a cause of back pain. $A m \mathcal{F}$ Roent 1935; 33: 209-13.

5 Ghormley R K. Low back pain with special reference to the articular facets with presentation of an operative procedure. $\mathcal{F} A M A$ 1933; 101: 1773 .

6 Rees W E S. Multiple bilateral subcutaneous rhizolysis of segmental nerves in the treatment of intervertebral disc syndrome. Ann Gen Pract 1971; 26: 126-7.

7 Toakley J G. Subcutaneous lumbar "rhizolysis"-an assessment of 200 cases. Med f Aust 1973; 2: 490-2.

8 Oudenhoven R C. Articular rhizotomy. Surg Neurol 1974; 2: $275-8$

9 Shealy C N. The role of the spinal facets in back and sciatic pain. Headache 1974; 14: 101-4. 
10 Pedersen H E, Blunck C F J, Gardner E. The anatomy of the lumbosacral posterior rami and meningeal branches of spinal nerves. f Bone foint Surg Am 1956; 38: 377-91.

11 Bradley K C. The anatomy of backache. Aust NZ f Surg 1974; 44: 227-32.

12 Bogduk N, Long D M. The anatomy of the so-called "articular nerves" and their relationship to facet denervation in the treatment of low-back pain. $\mathcal{F}$ Neurosurg 1979 ; 51: 172-7.

13 Nade S, Bell E, Wyke B D. The innervation of the lumbar spinal joint and its significance. $\mathcal{F}$ Bone foint Surg Br 1980; 62: 255 .

14 Putti V. New conceptions in the pathogenesis of sciatic pain. Lancet 1927; 2: 53-60.

15 Goldthwait J E. The lumbosacral articulation: An explanation of many cases of lumbago, sciatica and paraplegia Boston Med Surg $11911 ; 64: 365-72$.

16 Farfan H F, Huberdeau R M, Dubow H I. Lumbar intervertebral disc degeneration. The influence of geometrical features on the pattern of disc degeneration-a post mortem study. F Bone foint Surg Am 1972; 54: 492-510.

17 Putti V, Logroscino D. Anatomia dell'Artritismo Vertebrale Apofisario. Chir Organi Mov 1937/8; 23: 317-53.

18 Burk W. Ueber einen Bruch des Gelenkfortsatzes des 5. Lendenwirbels. Beitr Klin Chir 1908; 48: 558.

19 Koch K. Die isolierten Gelenkfortsatzbrüche der Lendenwirbelsaüle. Deutsche Z Chir 1923; 180: 339.

20 Mitchell C L. Isolated fractures of the articular processes of lumbar vertebrae. $\mathcal{F}$ Bone foint Surg 1933; 15: 608

21 Butler D, Trafimow J H, Andersson G B J, McNeill T W, Huckman M S. Discs degenerate before facets. Spine 1990; 15: 111-3.

22 Ingelmark B E. Function of, and pathological changes in the spinal joints, IV. Acta Anat Suppl 1959; 36: 12-57.

23 Lewin T. Osteoarthritis in lumbar synovial joints. A morphologic study. Acta Orthop Scand Suppl 1964; 73: 1-112.

24 Ziv I, Maroudas C, Robin G C, Maroudas A. The relationship between the degeneration of the human intervertebral disc and facet joints of the same lumbar segment-a physico-chemical study. 36th Annual Meeting of the Orthopedic Research Society, New Meeting of the Orthopedic Resear
Orleans, Louisiana February 5, 1990.

25 Meachim G. Light microscopy of Indian ink preparations of fibrillated cartilage. Ann Rheum Dis 1972; 31: 457-64.
26 Nachemson A L. Lumbar intervertebral pressure: Experimental studies on post-mortem material. Acta Orthop Scand Suppl 1960; 43: 42-3.

27 Dunlop R B, Adams A, Hutton W C. Disc space narrowing and the lumbar facet joints. F Bone foint Surg Br 1984 66: 706-10.

28 Smeathers J E, Joanes D N. Dynamic compressive properties of human lumbar intervertebral joints: A comparison between fresh and thawed specimens. $\mathcal{f}$ Biomech 1988; $21: 425-33$.

29 Malmivaara A, Videman T, Kuosma E, Troup J D G. Facet joint orientation, facet and costovertebral joint osteoarthrosis, disc degeneration, vertebral body osteophytosis and Schmorl's nodes in the thoracolumbar region of and Schmorl's nodes in the thoracolumb

30 Baker W C, Thomas G T, Kirkady-Willis W H. Changes in the cartilage of the posterior intervertebral joints after anterior fusion. $\mathcal{F}$ Bone foint Surg $B r$ 1969; 51: 736-46.

31 Parkkinen J J, Lammi M J, Ikonen J, Helminen $H$ Tammi $M$. The influence of cyclic hydrostatic pressure on cultured articular cartilage and chondrocytes. Abstract C18, Joint Destruction in Arthritis and Osteoarthrosis, 19th Symposium of the ESOA, Noordwijkerhout, Netherlands, 24-27 May 1992.

32 Urban J, Hall A. Physical modifiers of cartilage metabolism. In: Kuettner K, et al, eds. (Workshop Conference Hoechst Werk Kalle-Albert, Wiesbaden 1991.) Articular cartilage and osteoarthritis, ch. 27. New York: Raven Press, 1992; and osteoarth

33 Kahanovitz N, Bullough P, Jacobs R. The effects of internal fixation without arthrodesis on human facet cartilage. Orthop Trans 1983; 7: 14.

34 Swanepoel M W, Smeathers J E, Adams L M. The stiffnes of human apophyseal cartilage as an indicator of join loading. Proc Inst Mech Eng [H] 1994; 208: 33-43.

35 Seedhom B B, Takeda T, Tsubuku M, Wright V. Mechanical factors and patello-femoral osteoarthritis. Ann Rheum Dis 1977; 38: 307-16.

36 Bogduk N, Twomey L T. Movements of the lumbar spine In: Clinical anatomy of the lumbar spine, 2nd edn, ch. 7 . Edinburgh: Churchill Livingstone, 1991; 65-81.

37 Pearcy $M J$, Hindle $R$ J. Axial rotation of lumbar intervertebral joints in forward flexion. Proc Inst Mech Eng [H] 1991; 205: 205-9. 